\section{Recognition and management of lacunar strokes}

One in every five strokes results from injury to perforating arteries and arterioles in the deeper, non-cortical parts of the cerebrum and brain stem. ${ }^{1}$ The mechanism of these lesions remains controversial ${ }^{2}{ }^{4}$ : some are haemorrhagic but most present post mortem as small black dots ${ }^{5}$-lacunae-due to infarction and subsequent cavitation. Until the present decade virtually all lacunar strokes were associated with hypertension, ${ }^{3}$ but the proportion has now declined to around $70 \%$ with the other $30 \%$ due to thromboembolism or hypoperfusion from occlusive extracranial vascular disease. ${ }^{16} \mathrm{~A}$ few lesions $(35 \%)$ are asymptomatic but, because of their sensitive location, most $\left(65^{\circ} \mathrm{v}\right)^{7}$ present as transient ischaemic attacks with stepwise deterioration or as a sudden, severe clinical deficit. ${ }^{8}$ The discrete nature of the lesion is often suggested by the absence of visual field defects, seizures, loss of consciousness, coma, combined motor and sensory deficits, apraxia, aphasia, or agnosia. Angiography does not disclose the affected vessel and the electroencephalographic pattern remains non-focal. In general the prognosis for recovery is excellent, and prolonged rehabilitation is rewarding. ${ }^{8}$

The recognition that small lesions $(0.5$ to $20 \mathrm{~mm}$ diameter $)$ may produce lacunar syndromes has come from the careful correlation of clinical and anatomical data supported by relatively few necropsy studies. With successive improvements in computed tomography, the presence of a lacunar syndrome may be predicted where an acute neurological deficit, such as a pure motor hemiplegia, is accompanied by a normal CT scan. ${ }^{9}$ In some cases the scan can confirm the nature and location of a focal lesion. Nuclear magnetic resonance provides a similar degree of resolution but may prove more satisfactory for displaying infarction of the brain stem. ${ }^{10}$ With repeated computed tomography a positive scan is eventually obtained in more than two thirds of patients presenting with clinical syndromes. ${ }^{11}$ In the first week most infarcts are recognisable without contrast, but as the hypodense lesion becomes isodense contrast is needed to prevent "fogging." 12

In practical terms, neurologists can now diagnose clinically most haemorrhagic lesions, many infarcts, and about one fifth of lacunae. If the lesion is haemorrhagic or the patient hypertensive no further investigative procedures are indicated. If the patient is normotensive angiography should be considered only when extracranial arterial surgery is a practical possibility. ${ }^{13}$ In the future, however, the development of digital angiography and duplex scanning may be conducive to a more active approach. ${ }^{14}$

With increasing acceptance of the concept of lacunar syndromes a note of caution is inevitable. Many factors add to the hazards of diagnosis ${ }^{3}$ including the heterogeneity of presentation, incomplete syndromes in one third of those affected, ${ }^{11}$ variations in the nature of the lesions, and other manifestations of cerebrovascular disease such as superficial cortical infarction. A pure sensory stroke affecting the face, arm, and leg as a result of a lesion of the contralateral posteroventral thalamic nucleus may present with persistent numbness from the onset or with transient attacks leading to persisting numbness in only $10^{\circ}{ }_{0}$ of patients. Some patients may complain of paraesthesia, others of anaesthesia, dysaesthesia, or, occasionally, spontaneous thalamic pain. In Fisher's series $^{8}$ the CT scan was invariably negative.
With pure motor hemiplegia, the lesion is usually in the posterior limb of the internal capsule, but similar manifestations may arise from infarction of the basis pontis. Although the CT scan is usually positive, cortical somatosensory evoked responses after stimulation of the median nerve may indicate a sensory, lemniscal lesion in some patients. ${ }^{15}$ The close proximity of structures within the brain stem provides the setting for several lacunar syndromes. In the upper basis pontis the corticospinal fibres are dispersed into bundles, and lesions placed a few millimetres apart may cause either homolateral ataxia with crural paresis ${ }^{16}$ or facial weakness, severe dysarthria, and a clumsy hand. ${ }^{17}$ Lacunar strokes can produce Weber's syndrome of unilateral third nerve palsy with contralateral hemiplegia, Claude's syndrome of cerebellar ataxia and crossed third nerve palsy, hemiballismus from a lesion of the subthalamic nucleus of Luys, ${ }^{8}$ and bilateral lesions of the ventral pons in the locked in syndrome. ${ }^{18}$ Perhaps the most interesting symptom complex is the mesencephalothalamic syndrome resulting from an embolus at the top of the basilar artery-producing unilateral or bilateral third nerve palsies, paralysis of downward gaze, and drowsiness as a result of infarction in the territory of a perforating arteriole arising from the posterior cerebral artery. ${ }^{8}$

The impression that multi-infarct dementia results from repeated infarction-some symptomatic, some not-is probably erroneous. The initial lesion is almost always a symptomatic stroke, ${ }^{8}$ and more than three lacunar infarcts in one patient are rare. ${ }^{319}$ Dementia is usually associated with bilaterally distributed infarcts, ${ }^{20}$ but sudden impairment of memory may follow unilateral infarction of the fornix and corpus callosum, and dementia may result from an infarct in the central region of the thalamus or subthalamus. ${ }^{82}$ Other associated factors such as hypertension, ${ }^{22}$ non-occlusive arterial insufficiency, ${ }^{23}$ and normal pressure hydrocephalus ${ }^{824} 25$ frequently contribute to the clinical picture. The detection of lacunar infarcts by computed tomography has a practical value in the management of normal pressure hydrocephalus in that patients with this combination of diseases do not benefit from shunting. ${ }^{26}$

E M R CRITCHLEY

Consultant Neurologist,

Preston Infirmary,

Preston PR1 6PS

${ }^{1}$ Miller VT. Lacunar stroke: a reassessment. Arch Neurol 1983;40:129-34.

${ }^{2}$ Hughes W. Origin of lacunes. Lancet 1965;ii:19-21.

${ }^{3}$ Fisher CM. Lacunes: small, deep cerebral infarcts. Neurology 1965;15: 774-84.

+ Cole FM, Yates P. Intracerebral microaneurysms and small cerebrovascular lesions. Brain 1967;90:759-67.

5 Alvarez WC. Little strokes. Philadelphia: Lippincott, 1966.

${ }^{6}$ Fisher CM. Capsular infarcts: the underlying vascular lesion. Arch Neurol $1979 ; 36: 65-73$.

7 Kinkel PR, Kinkel WR, Jacobs L. Clinical-computerized tomographic (CT) correlations of cerebral lacunes. Neurology 1980;30:445.

${ }^{8}$ Fisher CM. Lacunar strokes and infarcts: a review. Neurology $1982 ; 32$ : 871-6.

${ }^{9}$ Harrison MJG. The investigation of strokes. In: Russell RWR, ed. Vascular disease of the central nervous system. 2nd ed. Oxford: Churchill, 1983:154-68.

" Bydder GM, Steiner RE, Young IR, et al. Clinical NMR imaging of the brain: 140 cases. AfR 1982;139:215-36.

11 Donnan GA, Tress BM, Bladin PF. A prospective study of lacunar infarction using computerized tomography. Neurology $1982 ; 32: 49-56$.

12 Skriver EB, Olsen TS. Serial computed tomography in cerebral infarction, investigations in the acute, subacute and chronic state. Acta Neurol Scand 1982;66, suppl $91: 6$.

${ }^{13} \mathrm{McD}$ owell $\mathrm{FH}$. Indications and selection of patients for extracranial 
carotid artery surgery for stroke. In: Goldstein M, ed. Cerebrovascular disorders and strokes. New York: Raven Press, 1979:351-4. (Advances in Neurology. Vol 25.)

14 Zbornikova V, Akesson JA, Link $\mathrm{H}$. Non-invasive diagnosis of carotid artery lesions: comparison between directional Doppler, duplex scanner and angiography. Acta Neurol Scand 1982;65, suppl 90:162-3.

${ }^{15}$ Chokroverty S, Rubino FA. "Pure" motor hemiplegia. 7 Neurol Neurosurg Psychiatry 1975;38:896-9.

${ }^{16}$ Fisher CM. Ataxic hemiparesis. A pathologic study. Arch Neurol 1978; 35: $126-8$.

17 Fisher CM. A lacunar stroke: the dysarthria-clumsy hand syndrome. Neurology 1967;17:614-7.

18 Karp JS, Hurtig HI. "Lucked-in" state with bilateral midbrain infarcts. Arch Neurol $1974 ; 30: 176-8$.

19 Ferrand J. Essai sur l'hémiplégie des vieillards : les lacunes de désintégration cérébrale. Paris: Thèse No 193, 1902.

${ }^{20}$ Ladurner G, Iliff LD, Lechner H. Clinical factors associated with dementia in ischaemic stroke. 7 Neurol Neurosurg Psychiatry 1982;45: 97-101.

${ }^{21}$ Wallesch CW, Kornhuber HH, Kunz T, Brunner RJ. Neuropsychological deficits associated with small unilateral thalamic lesions. Brain 1983; $106: 141-52$.

${ }^{22}$ Hachinski VC, Lassen NA, Marshall J. Multi-infarct dementia: a cause of mental deterioration in the elderly. Lancet $1974 ; \mathrm{ii}: 207-9,10$.

${ }^{23}$ Anonymous. Lacunes. Br Med F 1970;i :251.

${ }^{24}$ Earnest MP, Fahn S, Karp JH, Rowland LP. Normal pressure hydrocephalus and hypertensive cerebrovascular disease. Arch Neurol $1974 ; 31: 262-6$.

${ }^{25}$ Koto A, Rosenberg G, Zingesser LH, Horoupian D, Katzman R. Syndrome of normal pressure hydrocephalus: possible relation to hypertensive and arteriosclerotic vasculopathy. $\mathcal{F}$ Neurol Neurosurg Psychiatry 1977;40:73-9.

${ }^{26}$ Shukla D, Singh BM, Strobos RJ. Hypertensive cerebrovascular disease and normal pressure hydrocephalus. Neurology 1980;30:998-1000.

\section{Compliance and cancer chemotherapy}

Compliance with drug treatment is a problem in many branches of medicine but is not widely recognised as such in clinical oncology. Lewis et al have recently sought to broaden the concept of compliance from the traditional one of adherence by the patient to a prescribed oral regimen. ${ }^{1}$ They cited the doctor's behaviour, the doctor-patient relationship, and the degree of psychosocial support available to the patient as important factors in determining how much of the intended dose was actually taken, and reviewed the data on the effect of these reductions in dose on outcome.

Assessment of individual compliance is limited by the lack of a simple urine test for most anticancer drugs and complicated by the frequent use of multidrug treatments. One study used assay of urinary 17-ketogenic steroids to determine compliance with treatment with prednisone in 52 patients with acute leukaemia. Some $30 \%$ were found to be noncompliant, and this figure rose to $60 \%$ in the adolescent subgroup. ${ }^{2}$ Most other studies of compliance in patients with cancer have used the less reliable questionnaire approach.

Nausea and vomiting are probably the most frequent symptoms contributing to non-compliance, and there have been several recent attempts to refine antiemetic treatment. ${ }^{3}$ The control of severe "central" nausea and vomiting is still, however, far from perfect, and treatment will continue to cause many patients substantial distress.

The extent to which physicians' attitudes to adherence to the prescribed dose affect the doses actually administered has been studied in several multicentre trials. In the Medical Research Council study on advanced ovarian cancer, the protocol was substantially modified or abandoned as a result of toxicity in $44 \%$ of 130 patients in the combination chemo- $\frac{\pi}{\mathbb{D}}$ therapy arm compared with only $14 \%$ of 131 patients in the $\frac{\varrho}{c}$ oral single agent arm. ${ }^{4}$ To these figures should be added the extent of non-compliance by the patient which could not be $\bar{J}$ assessed in that study. A review of the National Surgical ग0 Adjuvant Breast Project study showed that about $30^{\prime}$ " of the $\Phi$ patients failed to complete the two years of treatment. Furthermore, the variation in the incidence of gastrointestinal toxicity from institution to institution suggested substantial differences $\stackrel{\vec{S}}{\vec{P}}$ in the recognition of patients' complaints or the emphasis? given to them. ${ }^{5}$

Two studies from major cancer centres have looked at the $\frac{\overline{\bar{\omega}}}{\vec{D}}$ effects on outcome of the reduction in dose by physicians. $\stackrel{\unrhd}{\unrhd}$ Bonnadonna and Valagussa analysed the reductions in dose in 238 patients given adjuvant combination chemotherapy $\overrightarrow{0}$ for breast cancer. ${ }^{6}$ The five year survival of those who received more than $85 \%$ of the intended dose was $77 \%$ com- $\vec{\sigma}$ pared with $45 \%$ for those who received less than this dose level $(\mathrm{p}<0.0001)$. There was no observed relation between age and the reduction in dose due to toxicity. A more recent $\infty$ study from Stanford applied complex statistical analysis to ${ }^{\circ}$ data on 123 patients with Hodgkin's disease. ${ }^{7}$ The rate of ${ }_{0}^{\mathscr{E}}$ drug delivery during the first three cycles of combination? chemotherapy was found to be important in achieving a complete response. The rate of delivery of nitrogen mustard was the third most significant variable in predicting increased $\vec{\nabla}$ survival, after age and pleural disease.

In the past few years doctors have, rightly, become in- $\mathbb{D}$ creasingly concerned about the quality of life for patients $\frac{\mathbb{D}}{3}$ receiving cancer chemotherapy. Once the decision to treat has 0 been taken, however, and the aim clearly defined as cure or $\underline{\text { }}$ palliation, undue concern for safety or acceptability should $\vec{\oplus}$ not be allowed to obscure the principal goal of efficacy.. Review of the original decision or modification of the dose may be indicated more frequently in cases where treatment is given with palliative rather than curative intent. In humanō tumours neither the relation of dose to response nor its relation to toxicity is well understood, and reductions in dose $\varrho$ have to be based on early toxicity assessed weeks, months, or $\overrightarrow{\overrightarrow{0}}$ years before any beneficial effect may be measured. Without 3 doubt, however, the low therapeutic ratio of cancer treatments results in considerable variations in the way doctors and patients carry out a treatment policy-though most would accept the convention that in cancer treatment should usually be pushed to the limits of toxicity. ${ }^{8}$ In order that these limits may be more clearly defined, doctors who treat patients with. cancer should be prepared to document the extent of and $\frac{\mathrm{O}}{3}$ reasons for deviations from the particular treatment policy being followed. They should also be in a position to evaluate the defined end points of that treatment-usually response, $\stackrel{\sim}{\rightarrow}$ interval free of disease, and survival-in terms of the treat- $\frac{D}{3}$ ment actually given and the toxicity encountered.

JOHN A GREEN

Senior Lecturer in Medical Oncology,

Clatterbridge Hospital,

Merseyside L63 4LA

${ }^{1}$ Lewis C, Linet MS, Abeloff MD. Compliance with cancer therapy by $\frac{\mathbb{D}_{\mathbb{D}}}{\mathrm{d}}$ patients and physicians. Am 7 Med 1983;74:673-8.

${ }^{2}$ Smith SD, Rosen D, Trueworthy RC, Lowman JT. A reliable method for evaluating drug compliance in children with cancer. Cancer 1979; $43: 169-73$.

${ }^{3}$ Penta JS, Poster DS, Bruno S, Macdonald JS. Clinical trials with anti- 